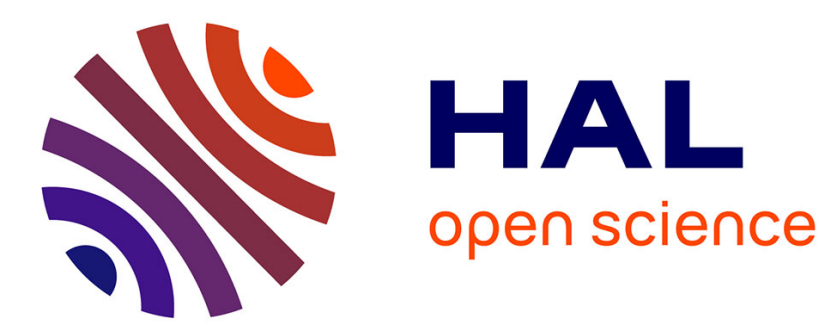

\title{
Simulation of Rain-Water Overland-Flow
}

Olivier Delestre, Stéphane Cordier, Francois James, Frédéric Darboux

\section{To cite this version:}

Olivier Delestre, Stéphane Cordier, Francois James, Frédéric Darboux. Simulation of Rain-Water Overland-Flow. 12th International Conference on Hyperbolic Problems, Jun 2008, College Park, United States. pp.537-546. hal-00343721

\section{HAL Id: hal-00343721 \\ https://hal.science/hal-00343721}

Submitted on 2 Dec 2008

HAL is a multi-disciplinary open access archive for the deposit and dissemination of scientific research documents, whether they are published or not. The documents may come from teaching and research institutions in France or abroad, or from public or private research centers.
L'archive ouverte pluridisciplinaire HAL, est destinée au dépôt et à la diffusion de documents scientifiques de niveau recherche, publiés ou non, émanant des établissements d'enseignement et de recherche français ou étrangers, des laboratoires publics ou privés. 


\title{
Simulation of Rain-Water Overland-Flow
}

\author{
O. Delestre, S. Cordier, F. James, and F. Darboux
}

\begin{abstract}
We are interested in simulating overland flow on agricultural fields during rainfall events. In this context, we definitely have to cope with dry/wet interfaces and water inflow on dry soil. The model considered is the Shallow Water system (or Saint-Venant equations) without infiltration. Moreover, the model has to be completed with friction terms.

It is numerically solved by a finite volume method. The topography and friction terms are treated by a well-balanced strategy with specific additional features to deal with dry/wet transitions.

The method as well as the choice of the friction term are validated on both analytical solutions and experimental dataset produced by INRA-Orléans.
\end{abstract}

\section{Introduction}

Rain on agricultural fields can yield to overland flow. This flow may have some undesirable effects. At the field scale, we can have soil erosion and pollutant transport. Downstream the fields, roads and houses may be damaged. To prevent these effects, control measures can be taken, such as grass strips. But we must know how the water is flowing in order to put efficiently these developments. This is exactly the context of the ANR project METHODE $[\mathbf{1 0}, \mathbf{1 1}]$ : the simulation of water flows on agricultural fields, thanks to the Shallow Water equations in the spirit of $[\mathbf{5}, \mathbf{6}]$.

This article is divided into two main parts. First we explain the numerical method for the resolution of the Shallow Water equations. We consider a hydrostatic reconstruction scheme coupled with two different treatments of the friction term. In the second part we describe two tests which allow us to validate the method: the first one is analytical and the second one is closer to the physical $20 \mathrm{C} 20$

1991 Mathematics Subject Classification. Primary 76M12, 74G05, 74G75; Secondary 35L65,

Key words and phrases. Shallow Water equations, finite volume schemes, well-balanced schemes, hydrostatic reconstruction, friction, rainfall hydrograph, analytical solution, dry/wet transitions.

This work was partially supported by the ANR grant "METHODE" \#ANR-07-BLAN-0232 and the Région Centre. The authors would like to thank Bernard Renaux and Loïc Prud'homme for the experimental setup, Marie-Odile Bristeau for fruitful discussions and Carine Lucas for her help and advice. 
problem we are interested in. At last we present numerical simulations which enable us to determine the most suitable method.

\section{Numerical method}

1.1. Numerical scheme. We consider the Shallow Water equations with topography

$$
\left\{\begin{array}{l}
\partial_{t} h+\partial_{x}(h u)=0 \\
\partial_{t}(h u)+\partial_{x}\left(h u^{2}+\frac{g h^{2}}{2}\right)=-g h \partial_{x} z
\end{array}\right.
$$

We use the second order hydrostatic reconstruction scheme proposed in $[\mathbf{1}, \mathbf{2}]$. It writes as:

$$
U_{i}^{n+1}=U_{i}^{n}-\frac{\Delta t}{\Delta x_{i}}\left(F_{i+1 / 2 L}^{n}-F_{i-1 / 2 R}^{n}-F c_{i}^{n}\right)
$$

with left and right numerical fluxes through interfaces:

$$
\begin{aligned}
& F_{i+1 / 2 L}^{n}=F\left(U_{i+1 / 2 L}^{n}, U_{i+1 / 2 R}\right)+\left(\frac{g}{2}\left(\left(h_{i+1 / 2-}^{n}\right)^{2}-\left(h_{i+1 / 2 L}^{n}\right)^{2}\right)\right), \\
& F_{i-1 / 2 R}^{n}=F\left(U_{i-1 / 2 L}^{n}, U_{i-1 / 2 R}\right)+\left(\frac{g}{2}\left(\left(h_{i-1 / 2+}^{n}\right)^{2}-\left(h_{i-1 / 2 R}^{n}\right)^{2}\right)\right),
\end{aligned}
$$

where $F$ is a consistent numerical flux for the Shallow Water system without source term, and the reconstructed states $U_{i+1 / 2 L}, U_{i-1 / 2 R}$ are defined by:

$$
\left\{\begin{array}{l}
U_{i+1 / 2 L}=\left(h_{i+1 / 2 L}, h_{i+1 / 2 L} u_{i+1 / 2-}\right), \\
U_{i-1 / 2 R}=\left(h_{i-1 / 2 R}, h_{i-1 / 2 R} h_{i-1 / 2+}\right), \\
h_{i+1 / 2 L}=\max \left(0, h_{i+1 / 2-}+z_{i+1 / 2-}-\max \left(z_{i+1 / 2-}, z_{i+1 / 2+}\right)\right), \\
h_{i-1 / 2 R}=\max \left(0, h_{i-1 / 2+}+z_{i-1 / 2+}-\max \left(z_{i-1 / 2-}, z_{i-1 / 2+}\right)\right),
\end{array}\right.
$$

We compute $U_{i+1 / 2-}$ and $U_{i-1 / 2+}$ with a MUSCL or an ENO reconstruction (see for example [3]). The values of $H_{i+1 / 2-}$ and $H_{i-1 / 2+}$ (where $H=z+h$ is the free surface level) are also reconstructed in order to get $z_{i+1 / 2-}$ and $z_{i-1 / 2+}$ for the case of dry/wet interface [1].

A cell-centered source term is added to preserve the consistency:

$$
\left.F c_{i}^{n}=\left(\begin{array}{c}
0 \\
\frac{g}{2}\left(h_{i-1 / 2+}^{n}-h_{i+1 / 2-}^{n}\right.
\end{array}\right) \Delta z_{i}\right)
$$

where $\Delta z_{i}=z_{i+1 / 2-}-z_{i-1 / 2+}$.

The second-order accuracy in time is recovered by the Heun method. We can write (1.1) under the form

$$
U^{n+1}=U^{n}+\Delta t \Phi\left(U^{n}\right)
$$

thus we get a second-order scheme in space and time

$$
\begin{aligned}
\hat{U}^{n+1} & =U^{n}+\Delta t \Phi\left(U^{n}\right), \\
\hat{U}^{n+2} & =U^{n+1}+\Delta t \Phi\left(U^{n+1}\right), \\
U^{n+1} & =\frac{U^{n}+\hat{U}^{n+2}}{2} .
\end{aligned}
$$

The boundary conditions are treated by the method of characteristics (see [4]). 
1.2. Numerical flux. We use the HLL flux [3]

$$
F\left(U_{l}, U_{r}\right)= \begin{cases}F\left(U_{l}\right) & \text { if } 0 \leq c_{1}, \\ \frac{c_{2} F\left(U_{l}\right)-c_{1} F\left(U_{r}\right)}{c_{2}-c_{1}}+\frac{c_{1} c_{2}}{c 2-c 1}\left(U_{r}-U_{l}\right) & \text { if } c_{1}<0<c_{2}, \\ F\left(U_{r}\right) & \text { if } c_{2}<0,\end{cases}
$$

with

$$
c_{1}=\inf _{U=U_{l}, U_{r}}\left(\inf _{j \in\{1,2\}}\left|\lambda_{j}(U)\right|\right), \quad c_{2}=\sup _{U=U_{l}, U_{r}}\left(\sup _{j \in\{1,2\}}\left|\lambda_{j}(U)\right|\right) .
$$

where $\lambda_{1}(U)=u-\sqrt{g h}$ and $\lambda_{2}(U)=u+\sqrt{g h}$ are the eigenvalues of the system.

1.3. Source terms treatment. We now consider the Shallow Water equations with rain $R(x, t)$ and friction $S_{f}$

$$
\left\{\begin{array}{l}
\partial_{t} h+\partial_{x}(h u)=R \\
\partial_{t}(h u)+\partial_{x}\left(h u^{2}+\frac{g h^{2}}{2}\right)=g h\left(S_{f}-\partial_{x} z\right) .
\end{array}\right.
$$

The rain is treated by a second-order accurate splitting

$$
U^{n+1}=S^{(\Delta t / 2)} S_{h}^{(\Delta t)} S^{(\Delta t / 2)}\left(U^{n}\right),
$$

where $S$ is the resolution of $\partial_{t} h=R$ and $S_{h}$ the application of the scheme (1.2).

Concerning the friction term, we use either Manning's friction law

$$
S_{f}=-\frac{k^{2} u|u|}{h^{4 / 3}}=-\frac{k^{2} q|q|}{h^{10 / 3}},
$$

or Darcy-Weisbach's friction law

$$
S_{f}=-\frac{k u|u|}{8 g h}=-\frac{k q|q|}{8 g h^{3}}
$$

where $k>0$ stands for the resistance value and $q=h u$ is the discharge. Both laws are derived from empirical considerations and are widely used in hydrology (see for instance $[\mathbf{5}, \mathbf{6}, \mathbf{8}, \mathbf{9}]$ ). This friction term has been treated by two different means (detailed in the following): the first one is the apparent topography method introduced in [3] and the second one is a semi-implicit treatment [4].

1.3.1. The apparent topography method. First, we should recall that the hydrostatic reconstruction has been designed in order to preserve steady states. Especially without friction term, the steady states at rest are:

$$
u=0, \partial_{x}(h+z)=0 .
$$

The key point of the apparent topography method, is to write the friction term as

$$
f_{r}(t, x)=S_{f}(h, u),
$$

so the steady states at rest are given by:

$$
u=0, \partial_{x}(h+z)=f_{r} .
$$

In this way we introduce a modified Shallow Water equation, with the apparent topography $z_{\text {mod }}=z-F_{r}$, where $\partial_{x} F_{r}=f_{r}$.

We apply the scheme (1.2) to this new topography. So we define $\Delta F_{r_{i+1 / 2}}^{n}=$ $f_{r i+1 / 2}^{n} \Delta x_{i+1 / 2}$ for the hydrostatic reconstruction and $\Delta F_{r_{i}}^{n}=f_{r_{i}}^{n} \Delta x_{i}$ for the cellcentered source term, with $\Delta x_{i+1 / 2}=x_{i+1}-x_{i}$ and $\Delta x_{i}=x_{i+1 / 2}-x_{i-1 / 2}$. 
We consider that there is no friction when there is no water. For Manning's law we take:

$$
f_{r i+1 / 2}^{n}= \begin{cases}0 & \text { if } h_{i+1 / 2-}^{n}=h_{i+1 / 2+}^{n}=0, \\ -\frac{k^{2} u_{i+1 / 2}^{n}\left|u_{i+1 / 2}^{n}\right|}{\left(h_{i+1 / 2}^{n}\right)^{4 / 3}} & \text { otherwise, }\end{cases}
$$

and for Darcy-Weisbach's law

$$
f_{r i+1 / 2}^{n}= \begin{cases}0 & \text { if } h_{i+1 / 2-}^{n}=h_{i+1 / 2+}^{n}=0 \\ -\frac{k u_{i+1 / 2}^{n}\left|u_{i+1 / 2}^{n}\right|}{8 g h_{i+1 / 2}^{n}} & \text { otherwise }\end{cases}
$$

with

$$
u_{i+1 / 2}^{n}=\frac{h_{i+1 / 2-}^{n} u_{i+1 / 2-}^{n}+h_{i+1 / 2+}^{n} u_{i+1 / 2+}^{n}}{h_{i+1 / 2-}^{n}+h_{i+1 / 2+}^{n}}
$$

and

$$
h_{i+1 / 2}^{n}=\frac{h_{i+1 / 2-}^{n}+h_{i+1 / 2+}^{n}}{2} .
$$

1.3.2. The semi-implicit treatment. We choose this method among other splitting methods not only because it preserves steady states at rest, but also for its stability. For Manning's law, following [4], we obtain

$$
q_{i}^{n+1}=\frac{q_{i *}^{n+1}}{1+g \Delta t \frac{k^{2}\left|u_{i}^{n}\right|}{\left(h_{i}^{n+1}\right)^{4 / 3}}}
$$

and for Darcy-Weisbach's law

$$
q_{i}^{n+1}=\frac{q_{i *}^{n+1}}{1+k \Delta t \frac{\left|u_{i}^{n}\right|}{8 h_{i}^{n+1}}},
$$

where $q_{i *}^{n+1}$ stands for the discharge obtained at each step of the Heun method $(1.2)$.

\section{Tests description and numerical validation}

2.1. Analytical solution test. Here we present briefly an adaptation to the 1-d case and our friction laws of an idea presented in $[\mathbf{8}, \mathbf{9}]$ for pseudo two dimensional cases. At steady states, we have $\partial_{t} h=\partial_{t} u=\partial_{t} q=0$, thus the massconservation equation gives $q=c s t$ and we get the equation

$$
\partial_{x} z=\left(\frac{q^{2}}{g h^{3}}-1\right) \partial_{x} h+S_{f}(q, h)
$$

where $S_{f}(q, h)$ depends on the friction law chosen (1.3) or (1.4). For any given value of the constants $k$ and $q$, once we are given an explicit expression for $h(x)$, then formula (2.1) allows us to compute the topography corresponding to this steady state and this water height. 
The configuration of our test is a channel of length $1 \mathrm{~km}$ (fig. 1). The water height $h(x)$ for the steady problem is given by

$$
h(x)=\left(\frac{4}{g}\right)^{1 / 3}\left(1+\frac{1}{2} \exp \left(-16\left(\frac{x}{1000}-\frac{1}{2}\right)^{2}\right)\right) .
$$

The flow is subcritical at inflow and outflow, so we impose a discharge of $q=2 \mathrm{~m}^{2} / \mathrm{s}$ at inflow and a water height corresponding to the value of $h(1000)$ downstream. The Manning roughness coefficient is $k=0.033$. The topography is calculated iteratively thanks to

$$
\partial_{x} z=\left(\frac{4}{g h(x)^{3}}-1\right) h^{\prime}(x)-\frac{4 k^{2}}{h(x)^{10 / 3}} .
$$

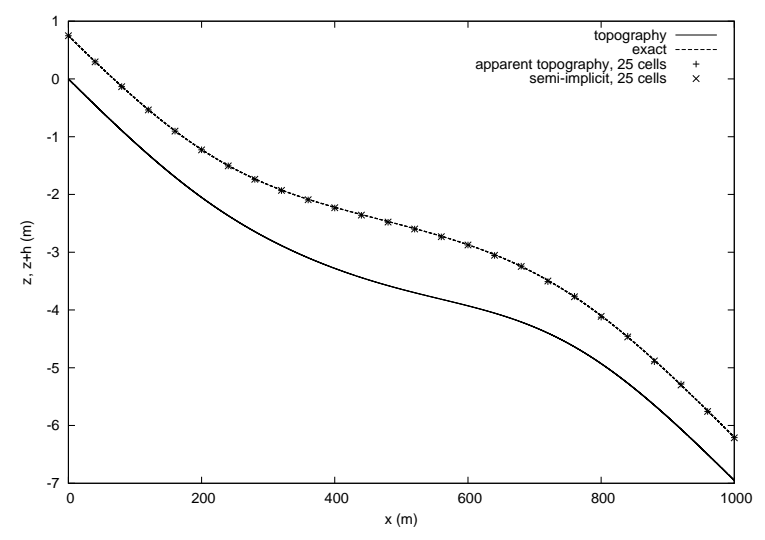

FiguRE 1. Steady state solution and numerical results.

The global relative error $E$ is defined as

$$
E=\sqrt{\sum_{i}\left(\frac{h_{i}^{n}-h_{i}^{e x}}{h_{i}^{e x}}\right)^{2}}
$$

where $h_{i}^{n}$ is the water height at the current time level and $h_{i}^{e x}$ the exact height. It turns out that both methods preserve correctly the steady state along time. However, since we are interested also in non-stationary solutions, we have considered an initially dry soil and the upstream discharge $q=2 \mathrm{~m}^{2} / \mathrm{s}$. Both methods (apparent topography and semi-implicit treatment) converge toward the steady state (fig. 2b). We show in (fig. 1) the free surface level at steady state. We can see that both methods give good results. In (fig. 1-a), we represent the discharge at equilibrium. Results are little better with the apparent topography method.

However, before the steady state is reached, we have a wet/dry transition (fig. 3b). We note that the apparent topography method is not adapted to this transition: we have a peak in the velocity that we do not get with the semi-implicit treatment (fig. 3-a). 



Figure 2. (a) Discharge comparison at steady state. (b) $L_{2}$ convergence in time.
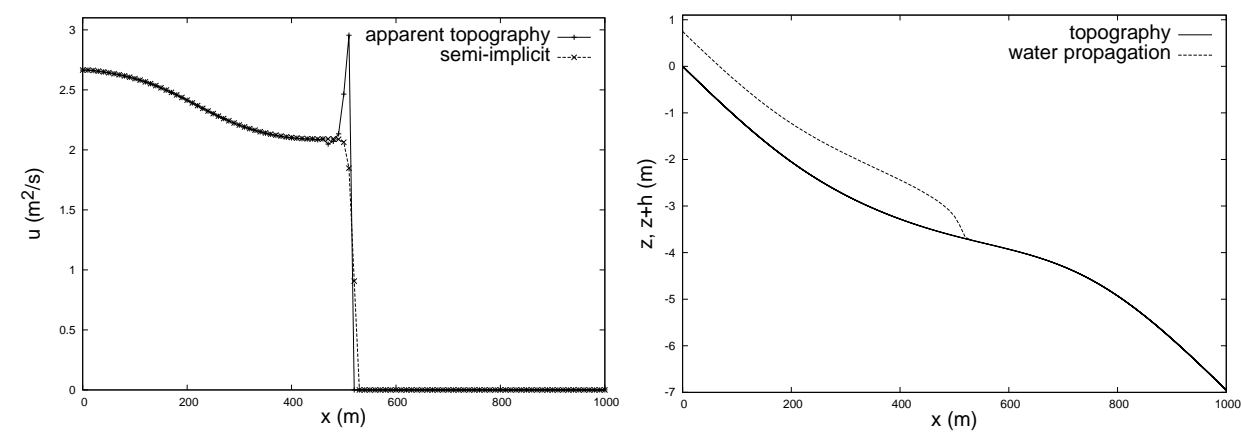

FiguRE 3. (a) Water front velocities comparison at $t=200 \mathrm{~s}$. (b) Water front propagation with the semi-implicit treatment at $t=$ $200 \mathrm{~s}$.

2.2. Rainfall hydrograph test. In this section we present another test case, based on experimental measurements realised thanks to the ANR project METHODE in a flume at the rain simulation facility at INRA-Orléans. The flume is $4 \mathrm{~m}$ long with a slope of $5 \%$ (fig. 4). The simulation duration is $250 \mathrm{~s}$. The rainfall intensity $R(x, t)$ is described by

$$
R(x, t)= \begin{cases}50 \mathrm{~mm} / \mathrm{h} & \text { if }(x, t) \in[0,3.95 \mathrm{~m}] \times[5,125 \mathrm{~s}], \\ 0 & \text { otherwise. }\end{cases}
$$

For this test, as there is no rain on the last $5 \mathrm{~cm}$, we have a wet/dry transition. The measured output is an hydrograph, that is a plot of the discharge versus time (fig. 6). The mathematical model for this ideal overland flow is the following: we consider a uniform plane catchment whose overall length in the direction of flow is $L$. The surface roughness and slope are assumed to be invariant in space and time.

We consider a constant rainfall excess such that

$$
R(x, t)= \begin{cases}I & \text { for } 0 \leq t \leq t_{d}, 0 \leq x \leq L, \\ 0 & \text { otherwise }\end{cases}
$$

where $I$ is the rainfall intensity and $t_{d}$ is the duration of the rainfall excess. First we compute some explicit "naive" analytical solution to the problem. We notice that three phases can clearly be identified on the hydrograph: a first non-steady step 

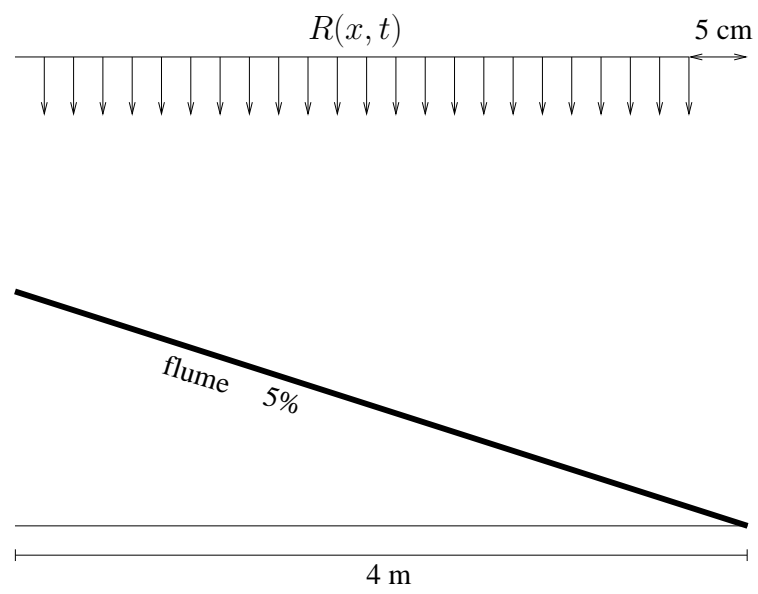

FigURE 4. Experimental configuration.

at the beginning of the rainfall event, then a steady-state and at last a non-steady step. The first and the second step solutions can be computed explicitly, and the "naive" solution is obtained by assuming a simple concatenation of the two parts.

As the rain begins to fall uniformly on the plane at $t=0$, we have $\partial_{x} h=\partial_{x} u=$ $\partial_{x} q=0$. So the local depth increases everywhere at the rate given by $\partial_{t} h=R(t)$. Since $I$ does not change in time, we have

$$
h=I t .
$$

This is the case initially, except at $x=0$ where there is no inflow from upstream. The local depth continues to grow everywhere according to (2.2) until the discharge reaches its limit, which must be equal to the inflow $q(x)=\int_{0}^{x} I d x=I x$ at time $t_{e q}(x)$. This is called the rising limb (fig. 5).

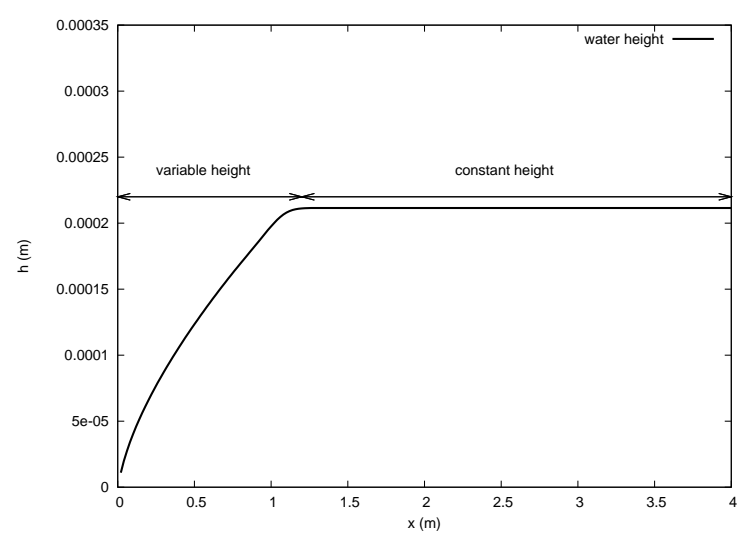

FiguRE 5. Water height during the rising limb, $t=25 \mathrm{~s}$.

Then the local depth and the local discharge remain constant. So when the equilibrium reaches the end of the plane at $x=L$, we get the peak flow and we 
have

$$
q(x)=I x, 0 \leq x \leq L,
$$

thus the second equation of the Shallow Water system writes

$$
\partial_{x}\left(\frac{q^{2}}{h}+\frac{g h^{2}}{2}\right)=g h\left(S_{f}(q, h)-\partial_{x} z\right) .
$$

After calculations with (2.3) and (2.4), we get

$$
\partial_{x} h=\frac{g h^{3}\left(S_{f}(x I, h)-\partial_{x} z\right)-2 x I^{2} h}{g h^{3}-x^{2} I^{2}},
$$

where we have for Manning's law

$$
S_{f}(x I, h)=-\frac{k^{2} I^{2} x^{2}}{h^{10 / 3}}
$$

and for Darcy-Weisbach's law

$$
S_{f}(x I, h)=-\frac{k I^{2} x^{2}}{8 g h^{3}} .
$$

Thus we are able to calculate the water height profile and the discharge for the times $0 \leq t \leq t_{d}$.

Now we compare at first numerical results with the analytical "naive" solution. Once again, with (fig. 6-a) we show that with the apparent topography method, we get a peak on the discharge downstream that we do not get far from this transition. With the semi-implicit method, we do not have this peak (fig. 6-b). This treatment gives good results closed to the naive exact solution. The hydrograph is well calculated (fig. 6-b), the height and the velocity too (fig. 7).
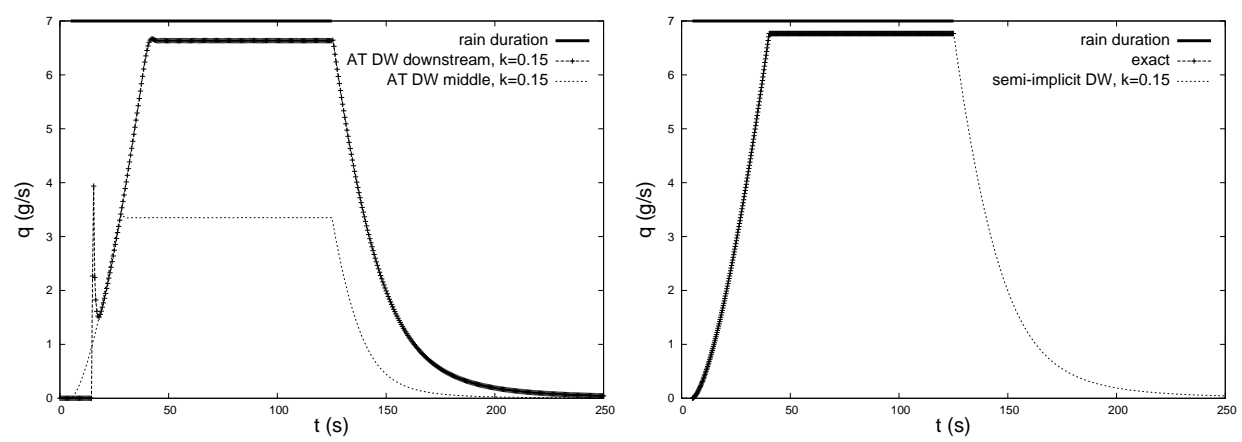

FiguRE 6. (a) Rainfall hydrograph with the apparent topography method (AT). (b) Rainfall hydrograph with the semi-implicit treatment for Darcy-Weisbach's law (DW).

Next, we propose a comparison between experimental measurements and numerical simulation (fig. 8), obtained with the Darcy-Weisbach's friction law. We obtain a reasonable agreement, but it turns out that it is impossible to fit correctly the shape of both the increasing and decreasing parts of the hydrograph. This indicates clearly that the model has to be modified, for instance by choosing alternative friction laws, but this is beyond the scope of this paper. 

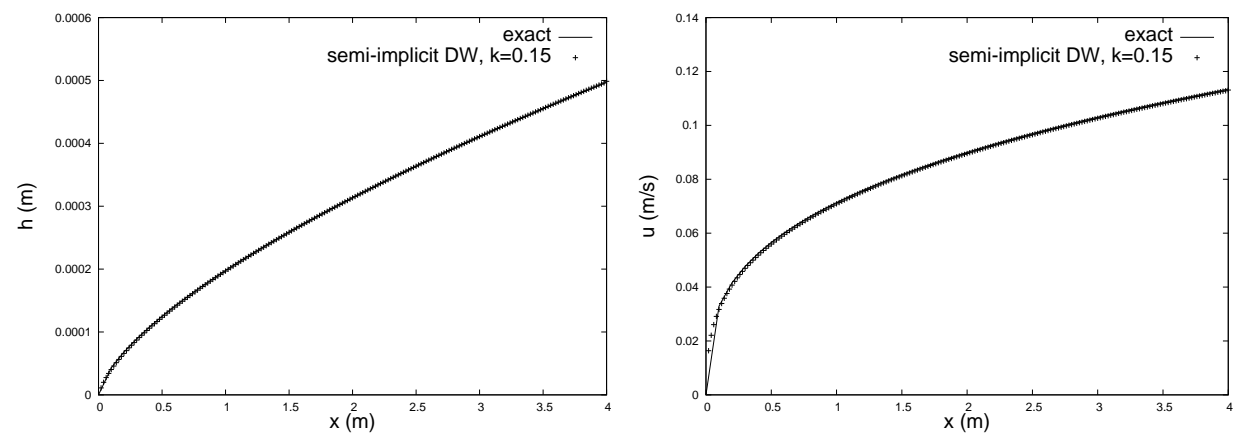

FIGURE 7. (a) Water height at steady state, $t=60 \mathrm{~s}$. (b) Velocity at steady state, $t=60 \mathrm{~s}$.

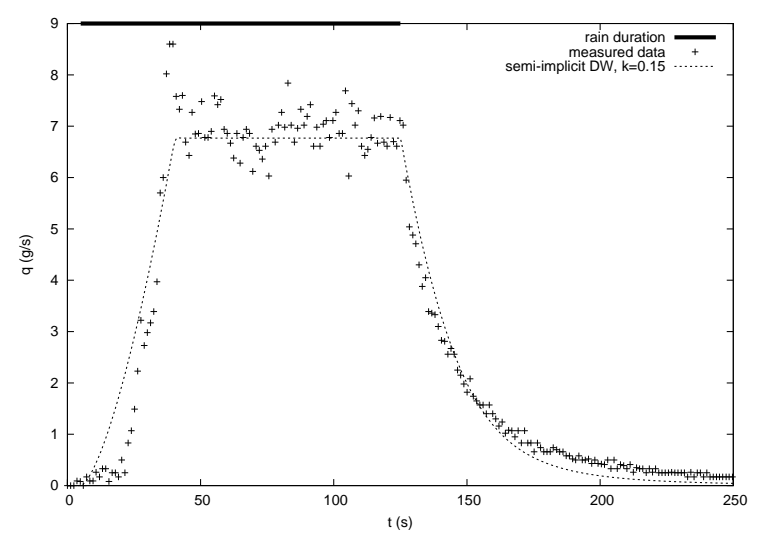

FiguRE 8. Comparison between experimental measures and numerical results.

\section{Conclusion}

This preliminary study of overland flow due to rainfall events clearly enlights several specific difficulties. First, from the numerical point of view, it seems that the apparent topography method, which was designed in order to catch steady states, is not adapted for wet/dry transitions. The semi-implicit treatment seems to be better in the problems we consider and gives good results compared to experimental data. Next, the model itself has to be improved, in particular regarding the empirical friction laws we used, which were not developed in this hydrological context. Finally, more realistic situations require infiltration and two-dimensional simulations, which are in progress and already validated on analytical solutions. This will be again compared with experimental data, as for the flume test.

\section{References}

[1] E. Audusse, F. Bouchut, M.-O. Bristeau, R. Klein, B. Perthame, A fast and stable wellbalanced scheme with hydrostatic reconstruction for shallow water flows, SIAM J. Sci. Comp., vol. 25, No. 6, 2004, pp. 2050-2065.

[2] E. Audusse, Modélisation hyperbolique et analyse numérique pour les écoulements en eaux peu profondes, Ph.D. Thesis, Université Paris VI, 2004. 
[3] F. Bouchut, Nonlinear stability of finite volume methods for hyperbolic conservation laws, and well-balanced schemes for sources, Frontiers in Mathematics, Birkhauser, 2004.

[4] M.-O. Bristeau, B. Coussin, Boundary conditions for the shallow water equations solved by kinetic schemes, Inria report, RR-4282, 2001.

[5] M. Esteves, X. Faucher, S. Galle, M. Vauclin, Overland flow and infiltration modelling for small plots during unsteady rain: numerical results versus observed values, J. Hydrol., vol. 228, 2000, pp. 265-282.

[6] F.R. Fiedler, J.A. Ramirez, A numerical method for simulating discontinuous shallow flow over an infiltrating surface, Int. J. Numer. Methods Fluids, vol. 32, 2000, pp. 219-240.

[7] J. M. Greenberg, A. Y. Leroux, A well-balanced scheme for the numerical processing of source terms in hyperbolic equation, SIAM Journal on Numerical Analysis, vol. 33, 1996, pp. 1-16.

[8] I. MacDonald, M.J. Baines, N.K. Nichols, P.G. Samuels, Steady open channel test problems with analytic solutions, Numerical Analysis Report, 2/95, Department of Mathematics, University of Reading, 1995, pp. 49-63.

[9] I. MacDonald, M.J. Baines, N.K. Nichols, P.G. Samuels, Analytic benchmark solutions for open channel flows, ASCE Journal of Hydraulic Engineering, vol. 123, No. 11, 1997, pp. 1041-1045.

[10] http://www.univ-orleans.fr/mapmo/methode/

[11] http://methode.netcipia.net/

MAPMO ORLÉANS, FRANCE

Current address: MAPMO, Université d'Orléans,, Route de Chartres, B.P. 6759 - 45067

Orléans cedex 2, FRANCE

E-mail address: olivier.delestre@etu.univ-orleans.fr

MAPMO OrlÉAns, France

E-mail address: stephane.cordier@univ-orleans.fr

MAPMO ORLÉANS, France

E-mail address: francois.james@univ-orleans.fr

INRA ORLÉANS, France

E-mail address: frederic.darboux@orleans.inra.fr 